\title{
Working Group on Extrasolar Planets
}

\author{
CHAIRPERSON: Alan P. Boss \\ MEMBERS: R. Paul Butler, William B. Hubbard, Philip A. Ianna, \\ Martin Kürster, Jack J. Lissauer, Michel Mayor, Karen J. Meech, \\ Francois Mignard, Alan J. Penny, Andreas Quirrenbach, \\ Jill C. Tarter, and Alfred Vidal-Madjar
}

Keywords. extrasolar planets

\section{Creation and Charge}

The Working Group on Extrasolar Planets (hereafter the WGESP) was created at a meeting of the IAU Executive Council in 1999 as a Working Group of IAU Division III and was renewed for three more years at the IAU General Assembly in 2003. The charge of the WGESP is to act as a focal point for international research on extrasolar planets. The membership of the WGESP has remained unchanged for the last three years.

\section{Definition of a "Planet"}

On February 28, 2003, the WGESP modified its position statement regarding the definition of a "planet" to the following:

Rather than try to construct a detailed definition of a planet which is designed to cover all future possibilities, the WGESP has agreed to restrict itself to developing a working definition applicable to the cases where there already are claimed detections, e.g., the radial velocity surveys of companions to (mostly) solar-type stars, and the imaging surveys for free-floating objects in young star clusters. As new claims are made in the future, the WGESP will weigh their individual merits and circumstances, and will try to fit the new objects into the WGESP definition of a "planet", revising this definition as necessary. This is a gradualist approach with an evolving definition, guided by the observations that will decide all in the end.

Emphasizing again that this is only a working definition, subject to change as we learn more about the census of low-mass companions, the WGESP has agreed to the following statements:

1) Objects with true masses below the limiting mass for thermonuclear fusion of deuterium (currently calculated to be 13 Jupiter masses for objects of solar metallicity) that orbit stars or stellar remnants are "planets" (no matter how they formed). The minimum mass/size required for an extrasolar object to be considered a planet should be the same as that used in our Solar System.

2) Substellar objects with true masses above the limiting mass for thermonuclear fusion of deuterium are "brown dwarfs", no matter how they formed nor where they are located.

3) Free-floating objects in young star clusters with masses below the limiting mass for thermonuclear fusion of deuterium are not "planets", but are "sub-brown dwarfs" (or whatever name is most appropriate).

These statements are a compromise between definitions based purely on the deuteriumburning mass or on the formation mechanism, and as such do not fully satisfy anyone on the WGESP. However, the WGESP agrees that these statements constitute the basis for 
a reasonable working definition of a "planet" at this time. We can expect this definition to evolve as our knowledge improves.

The above definition was accepted by IAU Division III at the Sydney General Assembly in August, 2003. In 2004, the IAU formed a Working Group on the Definition of a Planet (hereafter the WGDP), chaired by Division III President Iwan Williams. The WGDP has been actively discussing the low-mass end of the definition of a planet in the context of the known objects in our Solar System, without reaching a consensus as of the time of submission of this report (early September, 2005). The WGESP intends to accept the WGDP's definition of the low-mass end of the definition of a planet once an agreement has been reached.

\section{Conferences}

The WGESP supported the Bioastronomy 2004 meeting, held in Iceland in July, 2004 and organized by Commission 51. Because of the plethora of scientific meetings being held on the general subject of extrasolar planets, the WGESP has not felt the need to organize any further meetings on the subject, even at the upcoming General Assembly in Prague in August, 2006. Similarly, given the wealth of conference proceedings resulting from these meetings, the WGESP has not decided to create any publications about extrasolar planets. These decisions are largely a measure of the nearly universal excitement about the ongoing discoveries in this decade-old field, and of the strong support by the astronomical community and governmental funding agencies for this type of research.

\section{Lists of Planet Discoveries}

The WGESP maintains lists of extrasolar planetary candidates which are updated on a monthly basis. These lists are available from the WGESP web pages, which may be found at http://www.dtm.ciw.edu/boss/iauindex.html.

The criteria for inclusion in the WGESP lists include publication in a refereed journal and agreement by the WGESP that the discovery is likely to be correct. Doppler spectroscopy has accounted for nearly all of the discoveries to date, resulting in a lower limit on the planetary mass rather than a true mass. As a result, the WGESP has decided to list separately those candidate objects with minimum masses greater than 10 Jupiter masses, as such objects on average are likely to have true masses of 13 Jupiter masses or more, which would place them above the upper mass limit agreed upon by the WGESP (see above) for being a "planet".

\section{Highlights}

Essentially all of the planets discovered to date have been found by indirect detection techniques, primarily Doppler spectoscopy, and nearly all of these planets appear to be gas giant planets. The attainment of Doppler spectroscopy precisions of $\sim 1 \mathrm{~m} / \mathrm{s}$ has now allowed the lower mass limit to be extended to Neptune-mass and below, as shown by the discoveries of the planets orbiting GJ 436, Mu Arae, $\rho^{1}$ Cancri, and the 7.5 Earth-mass planet orbiting GJ 876. One or more of these may well be the first example(s) of one or two new classes of extrasolar planets, i.e., ice giant planets, or massive rocky planets.

The transit detection technique has now accounted for the discovery of six new planets, all confirmed by follow-up Doppler spectroscopy, with the first one being associated with the transiting event OGLE-TR-56 first observed by the Optical Gravitational Lensing 
Experiment (OGLE). Five of the six have been found by the OGLE survey, while the sixth (TrES-1) was found by the Transatlantic Exoplanet Survey (TrES).

A third indirect technique that has found a planet around a main sequence star is microlensing. The first microlensing detection was associated with the microlensing event known as OGLE 2003-BLG-235/MOA 2003-BLG-53, and recently a second event was followed, OGLE 2005-BLG-071. These two detections imply that giant planets are not rare around $\mathrm{M}$ dwarfs, the presumed host stars.

The very first planets were detected in orbit around a pulsar in 1991. While most subsequent searches for pulsar planets have been remarkably unsuccessful, a gas-giantplanet-mass companion to a binary star system containing a white dwarf and the pulsar PSR B 1620-26 in the M4 globular cluster has been detected using pulsar timing observations.

The Hubble Space Telescope accomplished the first characterizations of the atmosphere of an extrasolar planet by detecting the presence of several species $(\mathrm{Na}, \mathrm{H}, \mathrm{C}$, and $\mathrm{O}$ ) in the atmosphere of the transiting planet HD 209458b.

The field of extrasolar planets took another major step forward in 2005 with the announcement of several direct detections of extrasolar planets. The Spitzer Space Telescope accomplished the first direct detection of the light from two transiting planets, HD 209458b and TrES-1b, by measuring a difference in the total mid-infrared luminosity during the secondary eclipse of the planet by the star. The first image where an extrasolar planet is spatially resolved from the star appears to be that of the object orbiting the T Tauri star GQ Lup taken with the NACO adaptive optics system on the Very Large Telescope (VLT). The mass of the object is somewhat uncertain, however, and could exceed the 13-Jupiter-mass upper bound for being a planet. Prior to this discovery, the VLT imaged a roughly 5-Jupiter-mass companion to the brown dwarf 2M1207, itself with a mass of about 25-Jupiter-masses. This system thus has a mass ratio of $~ 5: 1$, typical of binary star systems. Nevertheless, the very low mass of the companion seems to place it in the planet category. The WGESP's definition of a planet notes that isolated planetary mass objects that have been imaged in regions of recent star formation should be referred to as "sub-brown-dwarfs" rather than planets, and the same logic may apply to $2 \mathrm{M} 1207 \mathrm{~b}$.

\section{Naming Planets?}

The WGESP has discussed the question of assigning names to extrasolar planets beyond the technical names currently assigned, e.g., HD 209458b, based on the name of the host star and the order in which the planets are discovered (b, c, d, ...). This approach is consistent with the IAU nomenclature system for multiple star systems. Given that some extrasolar planets are currently known only by lengthy appellations such as OGLE 2003BLG-235/MOA 2003-BLG-53, it is natural that journalists and the public would prefer in addition a more memorable name, similar to those of the objects in our Solar System. The WGESP is currently split on the question of whether or not the WGESP should consider the question of deciding upon informal names for extrasolar planet that would be in addition to the more technical, IAU-style names. Of those WGESP members who do think that the WGESP should decide upon informal names, there was a preference for these informal names to be chosen by the discoverers, subject to approval by the IAU (i.e., by the WGESP). Given the absence of a consensus opinion, however, the WGESP has tabled this issue for the moment, with the understanding that some discoverers may wish to proceed with suggesting names for their new extrasolar planets. 


\section{A New IAU Commission?}

A majority viewpoint of the WGESP is that the broad research areas encompassed by extrasolar planets deserve the creation of a new IAU Commission under Division III (Planetary Systems Science), while some WGESP members prefer that they be included in Commission 51 (Bioastronomy). The Division III Organizing Committee is contemplating a reorganization of their Division, including the creation of a new Commission on Extrasolar Planets. This would remove the need for having the WGESP. We recommend that the IAU move forward with resolving this key question at the 2006 General Assembly in Prague.

Alan P. Boss

Chairperson of the Working Group 